\title{
Salud bucal y calidad de vida en gestantes del Centro de Salud Cooperativa Universal febrero-julio 2016
}

Raquel Elizabeth Tolentino Valencia ${ }^{1, a}$

RESUMEN

Objetivo: Determinar la relación entre la salud bucal y la calidad de vida de las gestantes del Centro de Salud Cooperativa Universal.

Materiales y métodos: Estudio cuantitativo, observacional, descriptivo, transversal, retroprospectivo en una población de 116 gestantes durante los tres trimestres de gestación, en el periodo de febrero a julio de 2016, que asistieron al área de consulta externa odontológica del Centro de Salud Cooperativa Universal.

Resultados: El rango de edad materna más frecuente fue entre los 20 y 34 años (65,1\%), y el tercer trimestre de gestación fue el más frecuente (57\%). El 65,1\% de las gestantes presentaron un nivel regular de calidad de vida; el 20,9\%, un nivel bueno y el 14\%, una mala calidad de vida. El 87,2\% de las gestantes presentaron un nivel regular de higiene bucal con una prevalencia de caries del $11,2 \%$.

Conclusiones: La salud bucal y la calidad de vida de las gestantes presentaron una relación directa y moderada, debido a un nivel de higiene bucal regular y una alta prevalencia de caries dental. Ambas variables se encuentran estrechamente ligadas, demostrando la repercusión en su salud general y calidad de vida.

Palabras clave: Salud bucal; calidad de vida; gestantes (Fuente: DeCS BIREME).

\section{Oral health and quality of life in pregnant women at the Centro de Salud Cooperativa Universal during February-July 2016}

ABSTRACT

Objective: To determine the relationship between oral health and quality of life of pregnant women at the Centro de Salud Cooperativa Universal.

Materials and methods: A quantitative, observational, descriptive, cross-sectional, retrospective-prospective study of a population of 116 pregnant women during the three trimesters of pregnancy, from February to July 2016, who attended the dental outpatient department of the Centro de Salud Cooperativa Universal.

Results: The most frequent maternal age ranged from 20 to 34 years old $(65.1 \%)$, and the third trimester of pregnancy was the most common one (57\%). Sixty-five point one percent (65.1\%) of pregnant women had a fair quality of life, $20.9 \%$ had a good quality of life and $14 \%$ had a poor one. Eighty-seven point two percent (87.2\%) of pregnant women had a fair level of oral hygiene with a prevalence of cavities of $11.2 \%$.

Conclusions: Oral health and quality of life of pregnant women showed a direct and moderate relationship, due to a fair level of oral hygiene and a high prevalence of dental caries. Both variables are closely linked, demonstrating their impact on pregnant women's general health and quality of life.

Keywords: Oral health; quality of life; pregnant women (Source: MeSH NLM).

1. Cirujano Dentista, Maestra en Salud Pública y Gestión en Sistemas de Salud, Docente de pregrado.

a. Facultad de Estomatología, Universidad Inca Garcilaso de la Vega. Lima, Perú. 


\section{INTRODUCCIÓN}

La Organización Mundial de la Salud en 1990 informó que solo una de cada 10 personas tiene la totalidad de sus dientes sanos y obturados, por lo cual se considera a la caries dental como una enfermedad de carácter universal, que manifiesta su agresión desde el primer año de vida, afectando más o menos el $91 \%$ de la población mayor de cinco años de edad y siendo más frecuente entre las personas de cinco a catorce años de edad, en comparación con las enfermedades periodontales que ocurren con frecuencia en personas de mayor edad. Es decir, la caries dental constituye problemas graves de salud, no solo por su carácter mutilador, sino por las complicaciones sistémicas generadas ${ }^{(1)}$.

Es esencial que todos los individuos deban contar con una condición de salud bucal que les permita hablar, masticar, reconocer el sabor de los alimentos, sonreír, vivir libres de dolor e incomodidad y relacionarse con otras personas sin apremio ${ }^{(2)}$. Por lo tanto, al tener un estado de salud bucodental en pésimas condiciones, podría tener repercusiones en la salud general y en la calidad de vida de cada persona ${ }^{(3)}$. Cabe resaltar que no existe información actualizada con respecto a la salud bucal en gestantes, pero se debe tener en cuenta que la mujer durante el estado de gestación está sometida a una serie de cambios extrínsecos e intrínsecos relacionados entre sí que la hacen más vulnerable a la caries dental.

Además, la finalidad de los indicadores socio dentales o de calidad de vida es medir el nivel de satisfacción de las personas con su boca; así como su capacidad de normal funcionamiento, incluyendo tanto el funcionamiento físico, emocional y social ${ }^{(4)}$.

A nivel nacional nos encontramos que el Sistema Nacional de Salud Peruano tiene como finalidad que las instituciones que lo integran cumplan los roles coordinados y complementarios regidos por una política y estrategia común que asegure una atención de las prioridades sanitarias de nuestro país ${ }^{(5)}$.

La salud bucal en nuestro país constituye un problema de salud pública debido a la elevada prevalencia de caries dental en casi $90,4 \%$, según el estudio epidemiológico a nivel nacional realizado entre 2001-2002; según un estudio de 1990 tenemos una prevalencia de enfermedad periodontal del $85 \%$; y actualmente tenemos un $80 \%$ de maloclusiones en nuestra población, según estudios referenciales ${ }^{(6)}$. En 2000, en un estudio realizado por la Dirección General de Salud de las Personas, se encontró un $72 \%$ de prevalencia de enfermedad periodontal en gestantes ${ }^{(7)}$. En los datos estadísticos encontrados en la Dirección de Salud IV Lima Este de 2015, se encontró que 197 gestantes entre 12-17 años pasaron consulta odontológica y 2862 a partir de los 18 años en adelante; mientras que 1700 gestantes entre 12-17 años pasaron el examen odontológico y 22438 gestantes a partir de los 18 años. Muy pocas de estas gestantes recibieron algún tipo de tratamiento preventivo y se realizaron algún tratamiento recuperativo ${ }^{(8)}$.
Lo esencial en Salud Pública es promover la salud y prevenir las enfermedades, por lo que la estrategia de Salud Bucal como parte de la Dirección de Salud de las Personas tiene entre sus principales funciones la gestión de actividades promocionales, preventivas, recuperativas y de rehabilitación en todas las etapas de vida dentro del marco de atención integral de salud ${ }^{(7)}$. Por otro lado, el Seguro Social de Salud del Perú (EsSalud) recomienda que el cuidado de la higiene bucal debe iniciarse con las gestantes y recién nacidos, sobre todo llevar un chequeo odontológico preventivo durante el primer trimestre de gestación, lo cual puede asegurar un alumbramiento sin riesgos ${ }^{(9)}$.

A nivel del Centro de Salud Cooperativa Universal del distrito de Santa Anita, se encontró dentro de la información estadística proporcionada de 2015 que solamente 116 gestantes a partir de los 12 años de edad acudieron a los servicios de odontología para realizarle el examen respectivo y en muy pocas de ellas se realizaron las actividades recuperativas.

En cuanto al análisis de la morbilidad, las complicaciones del embarazo, parto y puerperio, así como las enfermedades dentales y de sus estructuras de sostén, han ido incrementando entre 2009 y $2011^{(5)}$. Por lo cual, la finalidad de la investigación fue encontrar la relación que existe entre la calidad de vida y la salud bucal en gestantes.

La investigación fue relevante porque se investigó un problema de salud pública, de interés político dentro de los programas institucionales para el manejo de la salud bucal de las gestantes y de interés institucional para el Centro de Salud Cooperativa Universal, del distrito de Santa Anita para fomentar actitudes favorables para el cuidado de la salud bucal de las gestantes, logrando estilos de vida saludables y por consiguiente una mejor calidad de vida. Además, la investigación fue transcendente, ya que los resultados obtenidos servirán para mejorar las estrategias sanitarias de salud bucal de las gestantes de la jurisdicción del Centro de Salud Cooperativa Universal del distrito de Santa Anita, ya que el objetivo en nuestro país es disminuir las enfermedades de la cavidad bucal, con la finalidad de mejorar la capacidad masticatoria de la población, autoestima y calidad de vida ${ }^{(10)}$.

El estudio fue factible, ya que se tuvo acceso a los recursos económicos, instrumentos validados y acceso a los datos como a los recursos humanos necesarios. Además, hubo un clima propicio para realizar la investigación en el establecimiento de salud del primer nivel de atención. Por otro lado, se contó con el permiso del jefe del centro de salud para realizar el estudio.

Finalmente, en este contexto nos planteamos como objetivo general determinar la relación entre la salud bucal y la calidad de vida de las gestantes; y como objetivos específicos conocer el estado de la salud bucal de las gestantes e identificar la calidad de vida de las gestantes del Centro de Salud Cooperativa Universal, Santa Anita, entre los meses de febrero a julio de 2016. 


\section{MATERIALES Y MÉTODOS}

El estudio realizado fue de enfoque cuantitativo, observacional, descriptivo, transversal y retroprospectivo. La población objetivo estuvo constituida por el total de gestantes que asistieron al área de consulta externa odontológica del Centro de Salud Cooperativa Universal, del distrito de Santa Anita entre los meses de febrero a julio en 2016. El universo poblacional estuvo constituido por 116 gestantes que cumplieron con el criterio de población objetivo, de las cuales se trabajó con una muestra calculada para estudios transversales de 86 gestantes, con un nivel de confianza del $95 \%$ y un error de $5 \%$, cuya selección fue por muestreo aleatorio simple.

Las participantes fueron gestantes durante los tres trimestres de gestación, que pasaron por un examen bucal respectivo y de manera voluntaria aceptaron llenar la encuesta de calidad de vida. Dentro de los criterios de exclusión, no se tomó en cuenta a todas aquellas gestantes que no contaban con el examen bucal respectivo (odontograma) e identificación de la placa dental.

La recolección de información se obtuvo a través de los hallazgos clínicos intrabucales registrados en el odontograma que formaban parte de la historia clínica de cada una de las pacientes del centro de salud para obtener el índice de caries CPO-D de la dentición permanente; así mismo, se recogió la información sobre el estado de su higiene bucal para medir su salud bucal. La información sobre la calidad de vida de las gestantes se obtuvo aplicando la encuesta del perfil del impacto de salud oral: OHIP-14 en su forma resumida de 14 preguntas, el cual es una medida genérica de la auto percepción del estado de salud de un individuo. Cada pregunta se subdividió en dos preguntas por cada una de las siete dimensiones.
El procesamiento de la información obtenida se organizó en una matriz de MS Excel para realizar su posterior análisis mediante el programa SPSS versión 17. Luego del análisis descriptivo se realizó el análisis de correlación entre las variables dependiente e independiente, mediante el coeficiente de correlación de Spearman p(rho), y los resultados obtenidos se representaron en tablas de acuerdo a los objetivos planteados en el presente trabajo.

\section{RESULTADOS}

De un total de las 116 gestantes que acudieron a consulta externa odontológica del Centro de Salud Cooperativa Universal, sólo se estudió a 86 gestantes que cumplieron con los criterios de inclusión. Con respecto a las características generales de las gestantes, se encontró que el rango de edad promedio fue entre los 20 a 34 años de 56 gestantes $(65,1 \%)$ y con relación a los trimestres de embarazo, el tercer trimestre fue de $49(57,0 \%)$ de las gestantes.

Al realizar la correlación entre las variables calidad de vida y salud bucal mediante la medida de correlación de Spearman, se encontró que esta fue altamente significativa, existiendo una relación directa y moderada entre las variables mencionadas. En relación entre la calidad de vida e índice de higiene oral de las gestantes se encontró un rho $=0,667 ; \mathrm{y}$ con respecto a los resultados de las dimensiones, los valores de rho Spearman no resultaron significativos para las dimensiones molestias psicológicas e incapacidad social (Tabla 1); de igual forma la relación entre calidad de vida e índice de caries (CPO-D) se encontró un rho $=0,516$ y con respecto a los valores de Rho Spearman, los resultados no resultaron significativos para la dimensión incapacidad social (Tabla 2).

Tabla 1. Correlación entre las variables calidad de vida e índice de higiene oral simplificado

$\begin{array}{llc}\text { Calidad de vida } & \text { Rho Spearman } & \text { Índice de higiene oral } \\ \mathbf{n}=86 & 0,667^{*} & \text { P-valor } \\ \text { (Dimensiones) } & & 0.000 \\ \text { Limitación funcional } & 0,786^{*} & \\ \text { Dolor físico } & 0,7 & 0.000 \\ \text { Molestias psicológicas } & -0,07 & 0.000 \\ \text { Incapacidad física } & 0,857^{*} & 0,52 \\ \text { Incapacidad psicológica } & 0,700^{*} & 0.000 \\ \text { Incapacidad social } & -0,021 & 0.000 \\ \text { Obstáculos } & 0,695^{*} & 0,851 \\ \text { Nivel de significancia: } \mathbf{0 . 0 5} & & 0.000\end{array}$


Tabla 2. Correlación entre las variables calidad de vida e índice de caries

\begin{tabular}{lll}
\multicolumn{1}{c}{ Calidad de vida } & Índice de caries & p-valor \\
n $=86$ & $0,516^{*}$ & 0.000 \\
(Dimensiones) & & \\
Limitación funcional & $0,452^{*}$ & 0.000 \\
Dolor físico & $0,403^{*}$ & 0.000 \\
Molestias psicológicas & $0,350^{*}$ & 0.001 \\
Incapacidad física & $0,360^{*}$ & 0.001 \\
Incapacidad psicológica & $0,357^{*}$ & 0.001 \\
Incapacidad social & 0,099 & 0,366 \\
Obstáculos & $0,431^{*}$ & 0.000
\end{tabular}

Al identificar los niveles de calidad de vida de las gestantes, se observó que, de las 86 gestantes, 56 presentaron un nivel regular de calidad de vida (65,1\%); 18 (20,9\%) un nivel bueno y solo 12 (14\%) obtuvieron mala calidad de vida (Tabla 3 ).

Tabla 3. Calidad de vida y sus dimensiones según los niveles de percepción de las gestantes

\begin{tabular}{|c|c|c|c|c|c|c|}
\hline \multirow[t]{2}{*}{ Variable } & \multicolumn{2}{|c|}{ Bueno } & \multicolumn{2}{|c|}{ Regular } & \multicolumn{2}{|c|}{ Malo } \\
\hline & Frec & $\%$ & Frec & $\%$ & Frec & $\%$ \\
\hline Calidad de Vida & 18 & 20,9 & 56 & 65,1 & 12 & 14,0 \\
\hline Dimensiones & & & & & & \\
\hline Limitación funcional & 25 & 29,1 & 49 & 57,0 & 12 & 14,0 \\
\hline Dolor físico & 34 & 39,5 & 33 & 38,4 & 19 & 22,1 \\
\hline Molestias psicológicas & 36 & 41,9 & 38 & 44,2 & 12 & 14,0 \\
\hline Incapacidad física & 16 & 18,6 & 56 & 65,1 & 14 & 16,3 \\
\hline Incapacidad psicológica & 40 & 46,5 & 29 & 33,7 & 17 & 19,8 \\
\hline Incapacidad social & 37 & 43,0 & 35 & 40,7 & 14 & 16,3 \\
\hline Obstáculos & 26 & 30,2 & 54 & 62,8 & 6 & 7,0 \\
\hline
\end{tabular}


Al evaluar la salud bucal de las gestantes, se encontró que, del total de las gestantes estudiadas, 75 (87,2\%) presentaron un nivel regular de higiene bucal; $11(12,8 \%)$ un nivel bueno y ninguna gestante presentó un nivel malo de higiene bucal (Tabla 4). Además, presentaron una prevalencia de caires dental de $11,2 \%$ con $8,1 \%$ piezas dentales perdidas; $26,8 \%$ piezas dentales cariadas y 8,1 piezas dentales obturadas (Tabla 5).

Tabla 4. Índice de higiene oral de las gestantes

\begin{tabular}{|ccccccc}
\multicolumn{1}{c}{ Salud bucal } & \multicolumn{2}{c}{ Bueno } & \multicolumn{2}{c}{ Regular } & \multicolumn{2}{c}{ Malo } \\
& Frec & $\%$ & Frec & $\%$ & Frec & $\%$ \\
\hline Índice de higiene oral & 11 & 12,8 & 75 & 87,2 & 0 & 0,0
\end{tabular}

Tabla 5. Prevalencia del índice de caries de las gestantes

\begin{tabular}{cc} 
Salud bucal & Prevalencia \\
Índice de caries & 11,2 \\
Piezas perdidas & 8,1 \\
Piezas cariadas & 26,8 \\
Piezas obturadas & 8,1 \\
\hline
\end{tabular}

\section{DISCUSIÓN}

En el presente trabajo de investigación se buscó principalmente determinar la relación entre la salud bucal y la calidad de vida de las gestantes. Conocer el estado de su salud bucal e identificar su calidad de vida. Se encontró que los resultados fueron altamente significativos en la muestra estudiada de 86 gestantes que formaron parte de la población asignada al Centro de Salud Cooperativa Universal en 2016. Se notó que la principal preocupación de las gestantes era realizar sus controles prenatales, dejando de lado su salud bucal. Se utilizó variables como: salud bucal, calidad de vida, edad materna y tiempo de gestación.

Al respecto Dobarganes et al., en su estudio sobre intervención educativa en salud bucal para gestantes en la Clínica Estomatológica Ignacio Agramonte del municipio de Camagüey, al igual que en este trabajo, utilizaron una muestra de 60 gestantes; Marrero et al., en su trabajo sobre diagnóstico educativo en salud bucal para gestantes trabajaron con una muestra de 55 gestantes y Bastarrechea et al., en su trabajo sobre la embarazada y su atención estomatológica integral como grupo priorizado atendidas en el área de Moncada trabajaron con una muestra de 33 gestantes; siendo este punto importante para conocer el tamaño de muestra con el que se trabaja en centros de salud del primer nivel de atención primaria ${ }^{(11-13)}$.

Como diferencia se puede agregar que en otros trabajos de investigación el tamaño de la muestra fue de mayor rango. Hinostroza en su tesis calidad de vida relacionada a salud oral uso el índice de salud oral general (GOHAl) en gestantes que acudieron al Hospital Nacional Cayetano Heredia, cuya muestra fue de 292 gestantes; Rivas et al., en su estudio sobre impacto de medidas preventivas sobre la calidad de vida de la salud bucodental en la gestante trabajaron con una muestra de 240 gestantes y Garbero et al., en su tesina salud oral en embarazadas trabajaron con una muestra dividida en dos grupos, 258 embarazadas correspondientes a los sectores privado y de la seguridad social del sistema de salud y 126 mujeres correspondientes del sector público (14-16).

Hinostroza en su tesis también consideró variables como: edad, calidad de vida y nivel educativo, encontrando que el rango de edad más frecuente fue entre los 25-29 años $(26 \%)^{(14)}$; Marrero et al., encontraron que su grupo etario predominante fue entre los $20-24$ años $(30,9 \%)$ seguido del rango entre los 25 a 29 años $(29,1 \%)^{(12)}$; Garbero et al., en su trabajo utilizó variables como: edad, nivel educacional, estado civil, trimestres de gestación, entre otros, obteniendo que la edad media del primer grupo fue de 23 años y del segundo grupo fue de 19 años, y la mayoría estaban en el segundo o tercer trimestre de gestación ${ }^{(16)}$ y Sotomayor et al., en su trabajo de investigación nivel de conocimiento en prevención de salud bucal en gestantes que se atienden en dos hospitales nacionales peruanos demostró que la edad más frecuente se encontró entre los 20 a 30 años $(50,4 \%)^{(17)}$.

Los resultados de dichas investigaciones concuerdan con los obtenidos en este estudio con una frecuencia de rango de edad materna mayor entre los 20 a 34 años, lo que refleja que dicho grupo etario se utiliza como grupos controles en otros tipos de investigaciones.

Respecto a los trimestres de gestación, Bastarrechea et 
al., mencionan que trabajaron con un grupo de gestantes en el tercer trimestre de gestación ${ }^{(12)}$; Estrada-Mendiola et al., en su trabajo evaluaron a las gestantes adolescentes en los diferentes trimestres de gestación ${ }^{(18)}$ y Carrión en su tesis importancia del control odontológico prenatal en las madres embarazadas que acuden al área de salud número 1 de la ciudad de Loja utilizó variables como: edad gestacional, edad de las gestantes, controles odontológicos, entre otros, mencionando que evaluó a su universo en los diferentes periodos de gestación, teniendo que acudieron con mayor frecuencia las gestantes entre las 20-30 semanas de gestación $(57 \%)^{(19)}$.

En el presente estudio se encontró que acudieron con mayor frecuencia al centro de salud en el último trimestre de gestación (57\%), esto debido a que durante el primer trimestre presentan mayor cantidad de náuseas, vómitos por reflujo, cambios en la dieta diaria entre otros, acudiendo al servicio de odontología durante el segundo o tercer trimestre de gestación.

En este estudio se analizó la calidad de vida de las gestantes utilizando el cuestionario del perfil del impacto de salud oral en su forma resumida de 14 preguntas, evaluando siete dimensiones, en donde se identificó la calidad de vida por niveles, encontrando un nivel regular en un $65,1 \%$ de las gestantes estudiadas, esto refleja la falta de conocimiento sobre las repercusiones de su salud general en su calidad de vida.

Al respecto, Hinostroza en su estudio obtuvo datos similares, pero utilizó el índice de salud oral general (GOHAI) para encuestar a las participantes, encontró que $80,1 \%$ de su muestra estudiada consideraba su calidad de vida baja, 19,2\% calidad de vida moderada y $0,7 \%$ calidad de vida alta ${ }^{(14)}$; Misrachi et al., en su trabajo sobre calidad de vida y condición de salud oral en embarazadas chilenas e inmigrantes peruanas realizado en una comuna de Santiago de Chile evaluaron la calidad de vida en salud oral mediante el cuestionario del perfil de impacto de salud oral, encontrando que el $33 \%$ consideraba mala su calidad de vida oral ${ }^{(20)}$ y EstradaMendiola et al., en su estudio utilizaron el instrumento de calidad de vida SF-36 que evalúa ocho dimensiones, encontró a 17,9\% gestantes en el primer trimestre de gestación, 37,5\% en el segundo trimestre; $44,6 \%$ y en el último trimestre, resultó con puntajes más bajos en todas las dimensiones ${ }^{(18)}$.

Respecto a la salud bucal de las gestantes, Carrión en su trabajo menciona que halló en su universo de estudio un alto porcentaje de caries dental (55\%), gingivitis $30 \%$ y enfermedad periodontal $15 \%{ }^{(19)}$; Bastarrechea et al., en su investigación encuentran en su muestra gingivitis $50 \%$, caries $21,2 \%$ y periodontitis $7,6 \%{ }^{(13)}$ y Dobarganes et al., en su estudio demuestra que su muestra presenta $76,6 \%$ de enfermedad periodontal y $61,6 \%$ caries dental ${ }^{(11)}$ y Marrero et al., en su investigación deduce que su muestra presentó enfermedad periodontal $(70,9 \%)$ y caries dental $(41 \%)^{(12)}$. Adicionalmente, Sotomayor et al., en su estudio reporta que $84 \%$ del total de todas las gestantes encuestadas no reportan atención odontológica. Estos resultados reflejan que la salud bucal constituye un problema de salud pública ${ }^{(17)}$.

La caries dental es muy frecuente durante el embarazo, debido a que durante esta etapa se encuentran sometidas a una serie de cambios, lo cual las hace más susceptibles de padecer la enfermedad, aunque la relación entre el embarazo y la caries dental aún no es bien conocida. Así tenemos que en la muestra estudiada presentó en cuanto a su salud bucal una prevalencia de $11,2 \%$ correspondiente al índice de caries y un nivel regular de higiene oral del total de las gestantes del presente estudio.

Los resultados obtenidos se asemejan con los encontrados por Misrachi et al., quienes hallaron un índice de caries de 10,22 sin diferencias significativa entre ambos grupos. Como diferencia se puede agregar, que en dicho trabajo el índice de higiene oral fue mejor en las embarazadas chilenas ${ }^{(20)} y$ García-Navas en su estudio calidad de vida oral en mujeres gestantes de la región de Murcia, no encontró diferencias estadísticamente significativas en cuanto al índice de caries total entre las mujeres embarazadas y el grupo control ${ }^{(21)}$.

Por otro lado, Laffita et al., en su trabajo de investigación salud bucal en gestantes de un área de salud urbana obtuvieron que las gestantes más afectadas fueron la de 20-34 años (34,4\%), seguidas por las de 25 a 29 años y en último lugar las gestantes entre los 30 a 34 años, hallando una predominancia de dientes obturados con un promedio de 6,3 dientes por mujer, dientes cariados 3,6 dientes por mujer y 3,4 dientes perdidos por mujer; y en cuanto a la de edad de 35 años a más, encontró 6,4 piezas dentales perdidas, siendo el índice de caries en este grupo etario de 13,3. En comparación con los resultados obtenidos en el presente estudio, se encontró una prevalencia de piezas dentales pérdidas de $8,1 \%, 26,8 \%$ piezas dentales cariadas y $8,1 \%$ piezas dentales obturadas ${ }^{(22)}$.

Dobarganes et al., en su trabajo encontró una higiene bucal deficiente en un $83,3 \%$ de las gestantes y $76,6 \%$ presentaron enfermedad periodontal, en contraste con los resultados obtenidos en la presente investigación se obtuvo que el nivel malo de higiene fue de $0,0 \%$ en las gestantes. Los resultados obtenidos reflejan la falta de conocimiento de parte de las gestantes sobre su salud bucal, por ello este estudio refleja la necesidad de un tratamiento recuperativo inmediato, una correcta enseñanza de la técnica de cepillado para la correcta eliminación de la placa dental durante esta etapa de vida para evitar enfermedades gingivales o periodontales ${ }^{(11)}$.

Además, se realizó la correlación entre las variables independiente y dependiente, encontrando una relación directa y moderada, según sus dimensiones, hallando que las dimensiones molestias psicológicas e incapacidad social no resultaron significativas.

Al respecto, Misrachi et al., en su estudio obtuvieron datos similares, encontrando una correlación significativa y directa solo del perfil de impacto de salud oral con el índice de caries en chilenas ${ }^{(20)}$. Como diferencia se puede agregar que Estrada-Mendiola et al., en su estudio obtuvieron puntajes más bajos en las dimensiones de rol físico y dolor corporal, y las dimensiones con menor alteración reportados por las pacientes fueron la función social, la función física y la salud general $^{(18)}$. 


\section{REFERENCIAS BIBLIGRÁFICAS}

1. Higashida Bertha Y. Odontología preventiva. 2 ed. México: Mc Graw-Hill. 2009.

2. Abanto J, Bönecker M, Raggio D. Impacto de los problemas bucales sobre la calidad de vida de niños. Rev Estomatol Herediana. 2010; 20(1):38-43.

3. Organización Mundial de la Salud. La OMS publica un nuevo informe sobre el problema mundial de las enfermedades bucodentales Comunicados de prensa 2004. Ginebra: OMS; 2004. [actualizada el 2017; acceso 11 de enero de 2017]. Disponible en: http://www.who.int/mediacentre/news/ releases/2004/pr15/es/

4. Misrachi C, Espinoza I. Utilidad de las medidas de la calidad de vida relacionada con la salud. Revista Dental de Chile. 2005; 96(2): 28-35.

5. Ministerio de Salud. Análisis de situación de salud del Perú. Lima: Dirección General de Salud de Epidemiología; 2013.

6. Ministerio de Salud. Salud Bucal. Lima: Dirección General de Salud de las Personas; 2015.

7. Ministerio de Salud. Documento técnico plan nacional de salud bucal "Sonríe Siempre Perú". Resolución Ministerial N516-2008/MINSA. Lima: MINSA; 2008.

8. Ministerio de Salud. Reporte C: actividades odontológicas. Estrategia Nacional de Salud Bucal. Lima: Dirección de Salud IV Lima Este; 2015.

9. EsSalud. Una mala salud bucal puede causar enfermedades cardiacas y pulmonares. Lima: EsSalud; 2014. [actualizada el 3 de noviembre de 2017; acceso 13 de enero de 2017]. Disponible en: http://www.essalud.gob.pe/essalud-unamala-salud-bucal-puede-causar-nfermedades-cardiacas-ypulmonares/

10. Ministerio de Salud. Gestión de la estrategia sanitaria nacional de salud bucal 2008-2011. Lima: Ministerio de Salud. Dirección General de las Personas. 2015

11. Dobarganes A, Lima A, López N, Pérez R, Gonzales L. Intervención educativa en salud bucal para gestantes. AMC. 2011; 15(3): 528-41

12. Marrero A, López E, Sayas S, Ribacoba E. Diagnóstico educativo en salud bucal para gestantes. AMC. 2004; 8(1): 104-14.

13. Bastarrechea M, Fernández L, Martínez T. La embarazada y su atención estomatológica integral como grupo priorizado. Area de salud Moncada. Rev Cubana Estomatol. 2009; 46 (4): 59-68.

14. Hinostroza D. Calidad de vida relacionada a salud oral usando el índice de salud oral general en gestantes que acuden al Hospital Nacional Cayetano Heredia. [tesis]. Lima: Universidad Peruana Cayetano Heredia. Facultad de Estomatología; 2016.

15. Rivas $M$, Romero $M$, De la Rosa $Z$. Impacto de medidas preventivas sobre la calidad de la salud bucodental en la gestante. Nure Inv. [Internet]. 2014 [citado 13 de enero de 2017]; 11(7). Disponible en: http://www.nureinvestigacion. es/OJS/index.php/nure/article/view/66/57
16. Garbero I, Delgado A, Benito de Cárdenas I. Salud oral en embarazadas: conocimientos y actitudes. Acta odontol. Venez. 2005; 43(2): 135-40

17. Sotomayor J, Reyes S, Ochoa J, Mallma A, Correa E, Arieta $\mathrm{J}$, et al. Nivel de conocimiento en prevención de salud bucal en gestantes que se atienden en dos hospitales nacionales peruanos. Odontol Sanmarquina. 2012; 15(1): 10-13.

18. Estrada-Mendiola R, Reynaga-Ornelas L, Jimenez-Garza 0 , Dávalos-Pérez A, Rodríguez-Lugo S. Diferencias en la calidad de vida por trimestre del embarazo en un grupo de adolescentes argentinas. Acta Universitaria. 2014;24(2): 57-60

19. Carrión N. Importancia del control odontológico prenatal en las madres embarazadas que acuden al área de salud nro. 1 de la ciudad de Loja durante el período de enero a julio del 2012. [tesis]. Ecuador: Universidad Nacional de Loja, Ecuador. Área de la Salud Humana; 2012.

20. Misrachi C, Ríos M, Morales I, Urzúa J, Barahona P. Calidad de vida y condición de salud oral en embarazadas chilenas e inmigrantes peruanas. Rev. Perú Med Exp Salud Pública. 2009; 26(4): 455-61.

21. García-Navas R. Calidad de vida oral en mujeres gestantes de la región de Murcia. [tesis]. España: Universidad de Murcia. Facultad de Medicina; 2015.

22. Laffita Y, Riesgo Y, Corujo C, Crespo M. Salud bucal en gestantes de un área de salud urbana. MEDISAN. [Internet]. 2009 [citado 13 de enero de 2017]; 3(4). Disponible en: http://www.bvs. sld.cu/revistas/san/vol13_4_09/san07409.htm

Fuentes de Financiamiento:

Este artículo ha sido financiado por la autora.

\section{Conflicto de Intereses:}

La autora declara no tener ningún conflicto de interés.

\section{Correspondencia:}

Raquel Elizabeth Tolentino Valencia

Dirección: Calle El Quetzal 148 Santa Anita, tercer piso, Lima-43, Perú. Teléfono: 511-958685653

Correo electrónico: raqueltolentinovalencia@outlook.com

Recibido: 08 de agosto de 2017 Evaluado: 10 de agosto de 2017 Aprobado: 04 de setiembre de 2017

(c) La revista. Publicado por Universidad de San Martín de Porres, Perú. (cc) ву Licencia de Creative Commons Artículo en acceso abierto bajo términos de Licencia Creative Commons Atribución 4.0 Internacional. (http://creativecommons.org/licenses/by/4.0/) 